\title{
A Commentary on Some of the Intrinsic Differences Between Grey Systems and Fuzzy Systems
}

\author{
Arjab Singh Khuman \& Yingjie Yang \\ Centre for Computational Intelligence \\ School of Computing \\ De Montfort University, \\ Leicester, United Kingdom
}

\author{
Robert John \\ Automated Scheduling, Optimisation \& Planning Group \\ School of Computer Science \\ University of Nottingham, \\ Nottingham, United Kingdom
}

\begin{abstract}
The aim of this paper is to distinguish between some of the more intrinsic differences that exist between grey system theory (GST) and fuzzy system theory (FST). There are several aspects of both paradigms that are closely related, it is precisely these close relations that will often result in a misunderstanding or misinterpretation. The subtly of the differences in some cases are difficult to perceive, hence why a definitive explanation is needed. This paper discusses the divergences and similarities between the interval-valued fuzzy set and grey set, interval and grey number; for both the standard and the generalised interpretation. A preference based analysis example is also put forward to demonstrate the alternative in perspectives that each approach adopts. It is believed that a better understanding of the differences will ultimately allow for a greater understanding of the ideology and mantras that the concepts themselves are built upon. By proxy, describing the divergences will also put forward the similarities. We believe that by providing an overview of the facets that each approach employs where confusion may arise, a thorough and more detailed explanation is the result. This paper places particular emphasis on grey system theory, describing the more intrinsic differences that sets it apart from the more established paradigm of fuzzy system theory.
\end{abstract}

\section{INTRODUCTION}

Uncertainty by its very nature is uncertain in its description, there is no notion of general uncertainty but rather, uncertainty that is context specific. Different facets of life will invoke different facets of uncertainty, it is only when the scope of the domain is somewhat known that a more informed approach can be undertaken. An understanding requires a perspective on which an assumption can be based. Defining a perspective provides the foundation on which to model, infer and reason from. Many approaches have been put forward as a means to tackle the various aspects of uncertainty, with each one taking a unique perspective; genetic algorithms (GA), neural networks (NN), particle swarm optimisation (PSO), principle component analysis (PCA), support vector machines (SVM) and so on. The very fact that there exists different models for uncertainty, reinforces the point that no one approach can be seen as a universal approach or a Jack of all trades. As the life and times of humanity progresses, so do the types of uncertainty that are associated. As a result, new paradigms may be proposed or existing paradigms maybe paired to create hybridised concepts. This paper will briefly describe two very prominent approaches, fuzzy system theory (FST) and grey system theory (GST). With the main aim being to provide for a readily accessible piece of work that clearly and concisely identifies the differences between the two.
The motivation for this paper comes from the lack of a precise understanding that describes the differences between the two concepts. Ergo, we believe that this paper in part helps with quashing some of the misinterpretations that can be associated with grey systems, via a clear, concise understanding of what actually sets the two approaches apart. In some instances, grey systems are generally considered to be an extension of fuzzy sets. This is true under certain considerations but not all, understanding when and where the differences occurs undoubtedly provides for a clearer picture. The close relationship between an interval-valued fuzzy set and a grey set is described. As is the subtly when describing the differences between interval representation and the generalised grey number. The added example based on preference analysis should also add the to the reader's understanding of what perspective each approach is adopting when tackling the problem of preference ordering. From two different schools of thought; the two paradigms are indeed very similar, however intrinsic differences still exist. The creation of this paper and the intended follow up papers will provide for a readily available, concise and detailed explanation of what differences exist between the two concepts.

Section II will go onto provide the foundational preliminaries for each approach, presenting some of the notation used. Section III will provide the observations, highlighting the differences and by proxy touching upon the similarities. Also included is an example of preference analysis done from a fuzzy and grey perspective. Section IV will draw out the conclusion and summarise upon the divergences that this paper has identified.

\section{PRELIMINARIES}

The paper will now go on to provide some of the core definitions needed in order to grasp the concepts being discussed.

\section{A. Fuzzy Preliminaries}

We begin with fuzzy theory and its associated extensions. The most fundamental aspect of fuzzy as with grey, is its understanding of numbers. A fuzzy number is ideal for describing linguistic phenomena, where an exact description of its state is unknown. For example, the following statement 'it's roughly 4pm' contains uncertainty, as allowances either side of 4 are included. This allows for a fuzzy number to be described in terms of a linguistic modifier, such as; nearly, 
almost, around and so on. In the literature there is often no distinction between a fuzzy number and a fuzzy interval, as both terms are used interchangeably. It can be stated that any interpretation involving a fuzzy view is an extension of a fuzzy number. This is true for a crisp number, an interval number, a number which is reference to about a point, or an interval which describes how near it is to a point. A fuzzy number and a fuzzy set are essentially the same, both mathematically and descriptively.

Definition 1 (Fuzzy set [8]): Let $U$ represent the universe and let $A$ be a set in $U(A \subset U)$. The fuzzy set $A$ is a set of ordered pairs given by the formal expression:

$$
\begin{gathered}
A=\left\{\left\langle x, \mu_{A}(x)\right\rangle: x \in U\right\} \\
A=\mu\left(x_{1}\right) / x_{1}+\mu\left(x_{2}\right) / x_{2}+\ldots+\mu\left(x_{n}\right) / x_{n}
\end{gathered}
$$

where $\mu_{A}: U \rightarrow[0,1]$ is the membership function and the value returned by $\mu_{A}(x)$ quantifies the belongingness of the element $x$ with respect to the fuzzy set $A$. In essence, the process of associating a membership value to an element is known as fuzzification. If $\mu_{A}(x)=1$, this signifies complete and absolute inclusion, whereas $\mu_{A}(x)=0$ signifies complete and absolute exclusion from the fuzzy set $A$. Any real value in the range $[0,1]$ signifies that it belongs in part to the set by some degree. A fuzzy set can also be represented by having an interval in place of a single crisp membership value, this is referred to as an interval-valued fuzzy set.

Definition 2 (Interval-valued fuzzy set [3]): Let $D[0,1]$ be the set of all closed sub-intervals of the interval $[0,1]$. $U$ represents the universe, $x$ is an element and belongs to the universe $x \in U$. An interval-valued fuzzy set is given by the formal expression:

$$
A=\left\{\left\langle x, M_{A}(x)\right\rangle: x \in U\right\}
$$

where $M_{A}: U \rightarrow D[0,1]$

The membership of an interval-valued fuzzy set itself is an interval. We omit the definitions for the various additional extensions to the standard fuzzy set; Atanassov intuitionistic, shadowed and type-2 fuzzy sets, as they are not compared against in this paper.

\section{B. Grey Preliminaries}

We will now present the preliminary concepts associated with grey systems, from which comparisons will be made. A grey set makes use of grey numbers $\mathrm{g}^{ \pm}$, and considers the characteristic function values of a grey set as grey numbers. There are several classes of grey numbers that the reader should be made aware of:

- $\quad$ Lower limit grey number: $\mathrm{g}^{ \pm} \in\left[\mathrm{g}^{-}, \infty\right)$

- $\quad$ Upper limit grey number: $\mathrm{g}^{ \pm} \in\left(-\infty, \mathrm{g}^{+}\right]$

- Interval grey number: $\mathrm{g}^{ \pm} \in\left[\mathrm{g}^{-}, \mathrm{g}^{+}\right]$

- $\quad$ Black number: $\mathrm{g}^{ \pm} \in\left(-\infty,{ }^{+} \infty\right)$
- White number: $\mathrm{g}^{ \pm} \in\left[\mathrm{g}^{-}, \mathrm{g}^{+}\right] \wedge \mathrm{g}^{-}=\mathrm{g}^{+}$

With the aforementioned classes one can interpret what is meant by white, black and grey. A white number is absolutely known and has an associated exact value. A black number is absolutely unknown, both in its exactness and the range in which it is to be found. A grey number is a halfway house between the two, where the exact value is unknown but the range in which it is to be found is known.

The generalised grey number presented in [5][6][7], is used in place of the standard grey number, as the allowance of both discrete and continuous values are perfectly acceptable. The notation that follows uses closed brackets, but its should be understood that both closed and open intervals are accepted.

Definition 3 (Generalised grey number [5][6][7]): Let $\mathrm{g}^{ \pm} \in \mathbb{R}$ be an unknown real number within a union set of closed or open intervals, presented as follows:

$$
\mathrm{g}^{ \pm} \in \bigcup_{i=1}^{n}\left[a_{i}^{-}, a_{i}^{+}\right]
$$

where $i=1,2, \ldots, n$ and $n$ is an integer value and $0<$ $n<\infty, a_{i}^{-}, a_{i}^{+} \in \mathbb{R}$ and $a_{i-1}^{-} \leq a_{i}^{-} \leq a_{i}^{+} \leq a_{i+1}^{-}$. For any interval $\left[a_{i}^{-}, a_{i}^{+}\right] \subseteq \bigcup_{i-1}^{n}\left[a_{i}^{-}, a_{i}^{+}\right], p_{i}$ is the probability for $\mathrm{g}^{ \pm} \in\left[a_{i}^{-}, a_{i}^{+}\right]$, if the following two conditions hold true:

- $\quad p_{i}>0$

- $\quad \sum_{i}^{n} p_{i}=1$

If so, $\mathrm{g}^{ \pm}$is a generalised grey number. $\mathrm{g}^{-}=\inf _{a_{i}^{-} \in \mathrm{g}^{ \pm}} a_{i}^{-}$ and $\mathrm{g}^{+}=\sup _{a_{i}^{+} \in \mathrm{g}^{ \pm}} a_{i}^{+}$are respectively referred to as the lower and upper limits of the grey number $\mathrm{g}^{ \pm}$. Based on this interpretation, it is impossible for there to be more than one number that is the underlying white number, contained within its candidate set. The generalised grey number is particularly important as it allows for the inclusion of both discrete and continuous datum to be contained in the candidate set. The very fact that both open and closed intervals may be included means that gaps may also be included, which would otherwise not be the case if using a standard interval representation.

Much like a fuzzy membership function value, a grey number $\mathrm{g}^{ \pm}$can also be attributed to a membership like value. In this instance the value is refereed to as the degree of greyness.

Definition 4 (Degree of greyness [4]): Quantifies the significance of the unknown grey number to the interval containing the information. The formal expression is given as:

$$
\mathrm{g}^{\circ}\left(\mathrm{g}^{ \pm}\right)=f\left(\mathrm{~g}^{-}, \mathrm{g}^{+}\right)
$$

where $f$ is a function to determine the significance of the grey interval to the grey number $\mathrm{g}^{ \pm}$. There are several variants for the degree of greyness, this paper will adopt the one presented in Eq. (5). Let $D=\left[d_{\min }, d_{\max }\right]$ be the domain of values represented by a grey number $\mathrm{g}^{ \pm} \in\left[\mathrm{g}^{-}, \mathrm{g}^{+}\right]$. We 
then have $d_{\text {min }} \leq \mathrm{g}^{-}, d_{\max } \leq \mathrm{g}^{+}$. We then can obtain the degree of greyness using the following expression:

$$
f\left(\mathrm{~g}^{-}, \mathrm{g}^{+}\right)=\frac{\left|\mathrm{g}^{+}-\mathrm{g}^{-}\right|}{\left|d_{\max }-d_{\min }\right|}
$$

As this is a measure for greyness of a grey number $\mathrm{g}^{ \pm}$, the value returned can be understood thusly:

- White number : $\mathrm{g}^{\circ}=0 \Leftrightarrow \mathrm{g}^{-}=\mathrm{g}^{+}$

- $\quad$ Black number : $\mathrm{g}^{\circ}=1 \Leftrightarrow \mathrm{g}^{-}=d_{\text {min }} \wedge \mathrm{g}^{+}=d_{\text {max }}$

- Grey number : $\mathrm{g}^{\circ}=[0,1] \Leftrightarrow\left\{\mathrm{g}^{-} \leq \mathrm{g}^{ \pm} \leq \mathrm{g}^{+}\right\}$

Understandably, if the degree of greyness is 0 then no uncertainty exists, meaning that the value is white, known and crisp. If the degree of greyness is 1 , absolute uncertainty exists and therefore deemed a black number. Any real value returned that falls in the range $[0,1]$ is a grey number.

In the same way a fuzzy set is an extension to the idea of a fuzzy number, a grey set is an extension to the idea of a grey number. The notion of grey sets themselves could also be described as either being; white, black or grey.

Definition 5 (White sets [5][6]): For a set $A \subseteq U$, if the characteristic function value for all $x_{i}$ elements with respect to $A$ can be expressed as crisp white numbers belonging to $v \in[0,1]$. The set $A$ can be assumed to be a white set.

Definition 6 (Black sets [5][6]): For a set $A \subseteq U$, if the characteristic function value for all $x_{i}$ elements with respect to $A$ can only be expressed as black numbers, then set $A$ can be assumed to be a black set.

Definition 7 (Grey sets [5][6]): For a set $A \subseteq U$, if the characteristic function value for all $x_{i}$ elements with respect to $A$ can be expressed by grey numbers, $\mathrm{g}_{A}^{ \pm} \in \bigcup_{i=1}^{n}\left[a_{1}^{-}, a_{1}^{+}\right] \in$ $D[0,1]^{ \pm}$. Where $D[0,1]^{ \pm}$is the set of all grey numbers within the interval $[0,1]$. Then set $A$ is assumed to be a grey set.

Similar to the expression of a fuzzy set as defined in Definition 1, the elements constituting the set are presented with their associated grey numbers:

$$
A=\mathrm{g}^{ \pm}\left(x_{1}\right) / x_{1}+\mathrm{g}^{ \pm}\left(x_{2}\right) / x_{2}+\ldots+\mathrm{g}^{ \pm}\left(x_{n}\right) / x_{n}
$$

The degree of greyness for an element belonging to a set can also be calculated, much like Definition 4, which presented the degree of greyness of the grey number with relation to its interval. The greyness measure for an element in relation to its set is presented as follows:

Definition 8 (Degree of greyness for an element [5][6]): Assume that $U$ is a finite universe of discourse and $x \in U$. For a set $A \subseteq U$, the characteristic function value of element $x$ with regards to $A$ is $\mathrm{g}_{A}^{\circ}(x) \in D[0,1]^{ \pm}$. The degree of greyness for an element is given by the following expression:

$$
\mathrm{g}_{A}^{\circ}(x)=\left|\mathrm{g}^{+}-\mathrm{g}^{-}\right|
$$

Based on the degree of greyness for an element, the degree of greyness for a set can also be computed, which is presented as follows:

Definition 9 (Degree of greyness for a set [5][6]): Assume $U$ is a finite universe of discourse, $A$ is a grey set such that $A \subseteq U$. Each $x_{i}$ element is with regards to the grey set $A$, $x_{i} \in U=i=1,2, \ldots, n$, where $n$ represents the cardinality. The degree of greyness of a set is given by the following expression:

$$
\mathrm{g}_{A}^{\circ}=\frac{\sum_{i=1}^{n} \mathrm{~g}_{A}^{\circ}\left(x_{i}\right)}{n}
$$

\section{OBSERVATIONS}

As grey numbers are predominantly associated with intervals, there is a very close relationship with interval-valued fuzzy sets. So much so that they are often regarded as being one and the same. Under certain considerations this is true, by using a grey set represented by only interval grey numbers an interval-valued fuzzy set is equivalent. There are some intrinsic differences between grey numbers and intervals, and grey sets and interval-valued fuzzy sets. If the perspective that a grey set and a interval-valued fuzzy set is equivalent, then one has ignored the possible inclusion of discrete data. Assume that the interval $[0.4,0.6]$ and the discrete set $\{0.4,0.6\}$ are two possible variances to represent a grey number $\mathrm{g}^{ \pm}$defined on $[0,1]$. As the first is an interval, there are an infinite number of possible candidates. The second variance, a discrete set, cannot be modelled using an interval, therefore having a different level of uncertainty associated with it. The generalised grey number, presented in Eq. (3), allows for the inclusion of all possible situations, whether discrete, continuous or a combination of both. It allows for potential gaps to be included and to be apart of the candidate set. For example, $\mathrm{g}^{ \pm} \in\{[1,4],[6,10]\}$ is a grey number where its underlying white number may belong to the interval $[1,4]$ or $[6,10]$. We know absolutely that it cannot be found between the values $(4,6)$. An interval encapsulating the same scope $[1,10]$ would include $(4,6)$ as possible candidates as it can not selectively ignore values inside the interval. If the grey number belonged to the candidate set $\mathrm{g}^{ \pm}=[1,10]$, then the values $(4,6)$ would be perfectly acceptable for both grey number and interval. The generalised approach could also be be used to represent a finite set of candidates, $\mathrm{g}^{ \pm}=\{2,4,6,8,10\}$, which is not representable by an interval. The dynamic nature of the generalised grey number far exceeds that of the interval in terms of encapsulation. The grey approach allows one to be selective, as to what should and should not be included in the candidate set. The following properties hold true for the generalised grey number [5]:

- $\mathrm{g}^{ \pm}$is a continuous grey number $\mathrm{g}^{ \pm} \in\left[a_{1}^{-}, a_{n}^{+}\right]$iff $a_{i}^{-}=a_{i-1}^{+}(\forall i>1)$ or $n=1$

- $\quad \mathrm{g}^{ \pm}$is a discrete grey number $\mathrm{g}^{ \pm} \in\left\{a_{1}, a_{2}, \ldots, a_{n}\right\}$ iff $a_{i}=a_{i}^{-}=a_{i}^{+}$

- $\quad \mathrm{g}^{ \pm}$is a mixed grey number iff it is made up of both crisp values and intervals

If there are no gaps between the candidate values, then a grey number and a interval are essentially the same. With the 
inclusion of gaps, the grey number is indeed different from the interval and an interval-valued fuzzy perspective.

\section{A. Sets}

Definition 5 defined a white set for a grey system, where for each value of $x$ with respect to its set $A$, its characteristic function value was a single white number. A type-1 fuzzy set can be regraded as a special instance of a white set. A crisp set is obviously a white set, and a crisp value is a white number; there is no associated uncertainty involved with either. A type1 fuzzy set will provide an accompanying membership degree for each and every element of its set, which is a white number and satisfies the definition for a white set. A white set has a clear and transparent relationship between its elements and the set itself. It should not be confused with the traditional interpretation of a crisp relationship. A element in a grey set, much like that of a standard fuzzy set, may be given a white number for its characteristic values. Replacing the grey characteristic function with that of a fuzzy membership function, a white set undoubtedly becomes a typical type-1 fuzzy set [5].

A fuzzy approach only deals with the notion of belongingness from a single perspective. A grey approach allows for three different variations; white black and grey, each with their own perspective. The is true not only for the number itself but also for sets, this is extended further to describe the elements of a set. Labeling an element as either being a white, black or a grey number with respect to its set, provides for a better understanding. Obviously the term white carries a connotation of completeness, fully knowing, but because of the existence of black and grey elements, relationships between elements and sets may be incomplete or unknown. A means to quantify this uncertainty due to incompleteness gave rise to the idea of a degree of greyness for a number, element and a set.

Assuming that $U$ is a finite universe of discourse and that $A$ is a grey set, $A \subseteq U$. Where $x$ is an element and $x \in U$, and the characteristic function value of $x$ is given by $\mathrm{g}_{A}^{ \pm}(x) . \mathrm{g}_{A}^{\circ}(x)$ is the degree of greyness of $\mathrm{g}_{A}^{ \pm}(x)$, and $\mathrm{g}_{A}^{\circ}$ is the degree of greyness for the grey set $A$. If and only if, $\mathrm{g}_{A}^{\circ}=0$ and $\mathrm{g}_{A}^{ \pm}(x) \in$ $[0,1]$ for any any $x \in U$, then it can be said that $A$ is a standard type-1 fuzzy set. Also, if and only if, $\mathrm{g}_{A}^{ \pm}(x)$ is a continuous grey number for any $x \in U$, then $A$ is an interval-valued fuzzy set. This enforces the the understanding that grey sets extend that of fuzzy and interval-valued fuzzy sets. Only when a grey number is represented by discrete or mixed sets, does the difference between an interval-valued fuzzy set become apparent.

A grey set will only be equivalent to an interval-valued fuzzy set when the characteristic function values are represented by interval grey numbers. Where the interval in the interval-valued fuzzy set is understood to be an unknown value within a known bounded scope [5].

\section{B. Degrees of uncertainty}

A fuzzy approach describes its element via the notion of a fuzzy membership function, assigning each element with a degree of membership $\mu_{A}\left(x_{i}\right)$. A value of 1 indicates absolute belongingness, 0 absolute exclusion, and $[0,1]$ partial inclusion to some degree, all with respect to its set. The degree of greyness of a grey number is concerned with relativity rather than absoluteness, the degree returned is based upon the limits of the grey number over the defined universe. To illustrate this point assume two grey numbers, $x^{ \pm} \in[25,75]$ and $y^{ \pm} \in\{25,75\}$, defined on the universe $[0,100]$. $x^{ \pm}$is an interval with an infinite number of possible candidates to choose from, $y^{ \pm}$contains two discrete numbers, meaning two possible choices. Using Eq. (5), the following degrees of greyness are returned for $x^{ \pm}$and $y^{ \pm}$:

$$
x^{ \pm}=\frac{75-25}{100-0}=0.5 \quad y^{ \pm}=\frac{75-25}{100-0}=0.5
$$

Both grey numbers return the same degree measure even though their cardinality are extremely different. This alludes to the fact that the degree is relative to the grey number itself, and not to the candidate set that it belongs. A degree of 1 has the opposite meaning to that of a fuzzy membership value, as a 1 according to grey systems indicates that absolute uncertainty exists and nothing is known. A degree of 0 means that the grey number has no uncertainty associated with it and everything regarding it is known. Using the degree of greyness to infer about a grey number can provide an understanding if the number is white or black. A value of 0 for the degree of greyness signifies that the number is white, and a value of 1 indicates that the number is in fact black.

\section{Preference analysis}

Preference analysis is the general understanding of group decision making policies. Inspecting the collective contributions of society, group or populous, to make an informed decision. Fuzzy preference involves the use of scoring; given a selection of criterion, experts are asked to score the criteria based on their opinions. A fuzzy approach allows for the use of multiple techniques to be incorporated. An individual could give their preference based on an ordered vector, $X=\left\{x_{1}, x_{2}, \ldots, x_{n}\right\}$, from best to worst. Utility values, providing a $U=\left\{x_{1}, x_{2}, \ldots, x_{n}\right\}$, where $x_{i} \in[0,1]$. In this case each alternative is scored based on a real value in accordance to the individual's preference. Given a preference based on the ordering vector, one can derive the utility values for the same domain. The intrinsic link between preference ordering $\Rightarrow$ utility values $\Rightarrow$ preference relations, allows for extreme versatility. A fuzzy preference relation is given by; $\mu_{p k}: X \times X \rightarrow[0,1]$, where $\mu p^{k}\left(x_{i}, x_{j}\right)=p_{i j}^{k}$ denotes the preference degree or the intensity of the alternative $x_{i}$ over $x_{j}$.

- $\quad p_{i j}^{k}=\frac{1}{2}:$ there is indifference between $x_{i}$ and $x_{j}$

- $p_{i j}^{k}=1: x_{i}$ is unanimously preferred to $x_{j}$

- $p_{i j}^{k}>\frac{1}{2}: x_{i}$ is preferred to $x_{j}$

A fuzzy preference relation is a matrix that can be intuitively read and inferred from. The use of the non-dominance concept further enhances the ability to understand which characteristic is preferred over another alternative, based on the opinion of experts [1].

A grey approach to preference analysis makes use of a system's incidence sequences. Even though traditional grey preference based relations do away with human experts, 
human opinion and expert knowledge can still be factored into the system [9]. For sake of simplicity we describe the traditional grey ethos to preference analysis. Grey systems takes into consideration the characteristic sequences of a system $Y_{1}, Y_{2}, \ldots, Y_{n}$, against its behavioural factor sequences $X_{1}, X_{2}, \ldots, X_{m}$, all of which must be of the same magnitude. In much the same way the fuzzy approached applied several techniques to quantify the data, grey preference analysis makes use of the degree of grey incidence, $\Gamma=\left[\gamma_{i j}\right]$. Where each entry in the $i^{\text {th }}$ row of the matrix is the degree of grey incidence for the corresponding characteristic sequence $Y_{i}$, and relevant behavioural factors $X_{1}, X_{2}, \ldots, X_{m}$. Each entry for the $j^{\text {th }}$ column is reference to the degrees of grey incidence for the characteristic sequences $Y_{1}, Y_{2}, \ldots, Y_{n}$ and behavioural factors $X_{m}$. One could also make use of the absolute matrix of grey incidence $A=\left[\epsilon_{i j}\right]_{n \times m}$. The relative matrix of grey incidence $B=\left[r_{i j}\right]_{n \times m}$, and the synthetic matrix of grey incidence $C=\left[p_{i j}\right]_{n \times m}$. Each variance of incidence takes a different perspective when inspecting the relationships between characteristics and behaviours, which like the fuzzy approach allows for additional versatility.

TABLE I. RECORDED VALUES FOR CHARACTERISTIC AND BEHAVIOURAL FACTORS

\begin{tabular}{|r|c|cccc|}
\cline { 3 - 6 } \multicolumn{1}{c|}{} & \multicolumn{4}{c|}{ Years } \\
\cline { 3 - 6 } \multicolumn{1}{c|}{} & 1984 & 1985 & 1986 & 1986 \\
\hline Production revenue: & $Y_{0}$ & 10,155 & 12,588 & 23,408 & 35,388 \\
\hline Fixed capitals: & $X_{1}$ & 3,799 & 3,605 & 5,460 & 6,982 \\
\hline Circulating capitals: & $X_{2}$ & 1,752 & 2,160 & 2,213 & 4,753 \\
\hline Labour forces: & $X_{3}$ & 24,186 & 45,590 & 57,685 & 85,540 \\
\hline After-tax profits: & $X_{4}$ & 1,164 & 1,788 & 3,134 & 4,478 \\
\hline
\end{tabular}

TABLE II. EXPERT PREFERENCE ORDERED VALUES

\begin{tabular}{|l|cccc|}
\cline { 2 - 5 } \multicolumn{1}{c|}{} & $X_{1}$ & $X_{2}$ & $X_{3}$ & $X_{4}$ \\
\hline Expert1: & 3 & 4 & 1 & 2 \\
\hline Expert2: & 4 & 3 & 1 & 2 \\
\hline Expert3: & 3 & 4 & 1 & 2 \\
\hline Expert4: & 4 & 3 & 1 & 2 \\
\hline Expert5: & 3 & 4 & 1 & 2 \\
\hline Expert6: & 3 & 4 & 1 & 2 \\
\hline
\end{tabular}

Table I contains real information regarding an economy that consists of non-governmental enterprises owned individually and collectively. The data was taken from enterprises based in the Henan Province of China, from the years 1984 through to 1986. The question being asked is, which factors should be given increased attention in order to maintain healthy growth for the coming years, so that $Y_{0}$ continues to trend positively?

We will first describe the grey process, $Y_{0}$ in this instance acts as the characteristics sequence, the sequences which is used to compare against the identified behavioural factors $X_{1}, X_{2}, \ldots, X_{4}$, in turn. The preference that will be identified is solely based on the data presented in Table I, there is no need to require the input of human intuition or experts. The majority of the working out has been omitted from this paper in order to save space, but assume that the zero starting point images for each $Y_{0}$ and $X_{i}$ have been established. At this point we could simply compute the degree of grey incidence, this would provide a general overview. However, the more typical thing to do given this situation is to compute the absolute degree of incidence $\left(\epsilon_{i j}\right)$ and the relative degree of incidence $\left(r_{i j}\right)$. From which the synthetic degree $\left(p_{i j}\right)$ of incidence can be computed. Each variation inspects the characteristic sequence in relation to each of the behavioural factors from a different perspective, based on the geometric shape of the sequence curves. As this is a rather trivial example with only one characteristic sequence, the returned matrices for absolute, relative and synthetic will all be $[1 \times 4]$. The absolute perspective inspects the characteristic and behavioural relationship in its entirety of absolute change, whereas the relative perspective inspects the relationship based on the data's initial starting points and relative rates of change. The synthetic perspective is a halfway-house between the two, which uses a variable coefficient $\theta \in[0,1]$ to stipulate how much of both to incorporate. Generally speaking, $\theta=0.5$, so that an equal amount of absoluteness and relativeness can be factored into the preference ordering. Assuming that the absolute and relative degree of incidence matrices have already been calculated, we use them to produce the synthetic degree of incidence using Eq. (8):

$$
p_{i j}=\theta_{\epsilon_{i j}}+(1-\theta) r_{i j}
$$

Which will result in the following $C=\left[p_{i j}\right]$ :

$$
C=\left[p_{i j}\right]_{1 \times 4}=\left[\begin{array}{llll}
0.6263 & 0.6618 & 0.7862 & 0.7355
\end{array}\right]
$$

The synthetic approach is often the one chosen as it employs qualities of both absolute and relative perspectives. Inspecting matrix $C$ we have the following order for preference; $p_{03}>p_{04}>p_{02}>p_{01}$. From which it can be concluded that:

$$
X_{3} \succ X_{4} \succ X_{2} \succ X_{1}
$$

Which states that $X_{3}$ is the most preferred, followed by $X_{4}$, then $X_{2}$, with $X_{1}$ being the least preferred factor. That is to say that labour forces have the greatest effect on production revenue $Y_{0}$, after-tax has the second greatest effect, followed by circulating capitals and finally fixed capitals. This ordering agreed very well with the actual situation of the region the data was based on. Echoing the same qualitative understanding that many human experts would have also given.

Using the fuzzy approach we must include the notion of human experts. The values contained in Table II are the preference ordering values given by six different experts. They were asked to order the importance of each of the behavioural factors contained in Table I, scoring them them in order of most important to least significant. For example Expert 1 identified factor $X_{3}$ as the most important and factor $X_{2}$ as the least important. It is noteworthy to mention that the characteristic factor $Y_{0}$ is not used. There is no need to utilise it in the same way as a grey approach, because the experts are providing their own assumptions in the ordering of preferences. The grey approach needed $Y_{0}$ to act as the characteristic sequence so that the geometric curve could be compared against each of the behavioural factors. Based on each of the experts' scores, a fuzzy preference relation must be created. Using the nondominance concept, we can derive an order for the factors, based on which is most strongly agreed with to the least agreeable. Assuming that $P$ is a fuzzy relation on $X, p_{i j}$ is the preference degree of $x_{i}$ over $x_{j}$. Whereas, $p_{j i}$ is the preference 
degree of of $x_{j}$ over $x_{i}$. If $p_{j i}-p_{i j}>0$ then we can say that $x_{i}$ is dominated by $x_{j}$ at degree $d\left(x_{i}, x_{j}\right)=\max \left\{p_{j i}-p_{i j}, 0\right\}$. We can also determine by what intensity $x_{i}$ is not dominated by $x_{j}$ at degree $1-d\left(x_{i}, x_{j}\right)$. The degree up to which $x_{i}$ is not dominated by any of the elements of $X$ is given by:

$$
\begin{aligned}
& \mu_{N D}\left(x_{i}\right)=\min _{x_{j} \in X}\left(1-d\left(x_{i}, x_{j}\right)\right)= \\
& 1-\max _{x_{j} \in X} d\left(x_{i}, x_{j}\right)
\end{aligned}
$$

The maximum non-dominated elements in $X$ is given by:

$$
X^{N D}=\left\{x_{i} \in X \mid \mu_{N D}\left(x_{i}\right)=\max _{x_{j} \in X} \mu_{N D}\left(x_{j}\right)\right\}
$$

The use of the average (AVG) operator was utilised to aggregate each indexed element for all six of the fuzzy preference relations, other t-norms are perfectly acceptable. Being the average, it lies directly between an optimistic lower bound and pessimistic upper bound, a halfway-house approach much like that of the synthetic degree of incidence when $\theta=0.5$. Averaging each indexed fuzzy preference relation matrix entry for all experts will produce a final single matrix from which we can derive the order of preference. Again we omit a considerable amount of working out in favour to present the final non-dominance degree matrix:

$$
\mathrm{ND}=\left[\begin{array}{llll}
0.00 & 0.00 & 0.78 & 0.44 \\
0.11 & 0.00 & 0.89 & 0.56 \\
0.00 & 0.00 & 0.00 & 0.00 \\
0.00 & 0.00 & 0.33 & 0.00
\end{array}\right]=\left[\begin{array}{l}
0.22 \\
0.11 \\
1.00 \\
0.67
\end{array}\right]
$$

Inspecting the $N D$ matrix and the with use of Eq. (9) we have the following order for preference; $p_{03}>p_{04}>p_{01}>$ $p_{02}$. From which it can be concluded that:

$$
X_{3} \succ X_{4} \succ X_{1} \succ X_{2}
$$

Interestingly enough, both the grey and fuzzy approaches identified that $X_{3}$ as the most important behavioural factor, followed by $X_{4}$. The difference between the two approaches came for the $3^{r d}$ and $4^{t h}$ positions. The fuzzy perspective using the AVG operator and non-dominance concept agreed that $X_{2}$ (circulating capitals) was the least significant. Whereas, the least significant factor according to a grey approach using the synthetic degree of incidence with $\theta=0.5$ was $X_{1}$ (fixed capitals). As this was based on a real example using historical data, the grey order of preference was better suited as it echoed more accurately what indeed was the case [4]. The striking similarities of the order of preference from both approaches given the fact that they were derived via different means, was to be expected. Granted, the example was trivial and small, but using the same ethos, both approaches will generally agree on the same preference. A fuzzy approach requires human interaction in order to encapsulate intuitiveness, whereas a grey approach concentrates solely on the underlying meaning of the data itself. With the addition of the various degrees of incidence one could use to generate the preference, the grey approach is more versatile than a standard fuzzy approach. Sometimes human expertise may be unavailable and therefore a grey approach will be more applicable. Grey systems are ideally suited where the data being used is minimal, grey forecasting can be done with a data sequence as small as four elements [2]. A grey approach can also be adapted to incorporate human expertise [9], making it even more versatile and adaptable to any given situation. This coupled with the fact that grey systems have reduced computational overhead, a grey approach will often be a good first choice [4].

\section{CONCLUSION}

This paper has set about describing some of the divergences between grey and fuzzy systems, in doing so it has also touched upon instances where similarity occurs. They are two distinct paradigms because they tackle the problem of quantifying and encapsulating uncertainty from different perspectives. As grey systems can be seen as an extension, or in specific cases a special interpretation of fuzzy systems, misunderstandings or misinterpretations can occur. The very foundations that the approaches themselves are built upon are different from one another; fuzzy is unclear intention clear extension, and grey is clear intention and unclear extension. The assumption that an interval-valued fuzzy set is the same for a grey number is only true when the notion of a grey number is understood as not being able to contain discrete data. As one can see from the generalised grey number presented in Definition 3, a grey number can indeed contain discrete data and a combination of continuous data. The additional robustness allows for the generalised approach to extend and even regress back to a standard interval-valued fuzzy set. Also described was how the concept of sets are understood from each approach. The difference in preference analysis from both paradigms was also discussed, identifying how each approach inspects the data to provide for an order of preference. The follow up to this paper will go into considerable more detail, outlining more in depth aspects of shared instances, with the overall goal of providing a detailed explanation of the divergences.

\section{REFERENCES}

[1] J. Fodor, M. Roubens. Fuzzy Preference Modelling and Multicriteria Decision Support. Dordrecht: Kluwer Academic Press (1994).

[2] Khuman, A.S.; Yingjie Yang; John, R., A New Approach to Improve the Overall Accuracy and the Filter Value Accuracy of the GM $(1,1)$ New-Information and GM $(1,1)$ Metabolic Models. Systems, Man, and Cybernetics (SMC), 2013 IEEE International Conference on , vol., no., pp.1282 - 1287, 13-16 Oct. (2013).

[3] R. Sambue. Fonctions $\Phi$ foues. Application I'Aide au Diagnostic en Patholologie Thyroidienne. PhD thesis, Univ. Marseille, France (1975).

[4] Liu Sifeng, Lin Yi. Grey Systems: Theory and Applications. Springer Verlag (2011).

[5] Yingjie Yang, Robert John. Grey sets and greyness, Information Sciences, Volume 185, Issue 1, 15 February 2012, pp. 249 - 264.

[6] Yang, Y, Liu, S, John, R. Uncertainty Representation of Grey Numbers and Grey Sets. Cybernetics, IEEE Transactions on, vol.PP, no.99, pp.1,1.

[7] Y. Yang. Extended Grey Numbers and Their Operations. In: Proceedings of 2007 IEEE International Conference on Systems, Man and Cybernetics, Montreal, Canada. pp. 2181 - 2186, October (2007).

[8] L.A. Zadeh. (1965), Fuzzy Sets. Information and Control. (8) pp. 338 353 (1965).

[9] Jijun Zhang, Desheng Wu, D.L. Olson. The method of grey related analysis to multiple attribute decision making problems with interval numbers. Mathematical and Computer Modelling. Volume 42, Issues 910, pp. 991 - 998, November (2005) 